\title{
Active control of the nonlinear bending behavior of magnetorheological elastomer sandwich beam with magnetic field
}

\author{
Nassim Zeerouni ${ }^{1}$, Salah Aguib ${ }^{2}$, Abdelkader Nour ${ }^{3}$, Toufik Djedid ${ }^{4}$, Ali Nedjar ${ }^{5}$ \\ Dynamic of Engines and Vibroacoustic Laboratory, F.S.I., M.B. Boumerdes University, \\ Boumerdes 35000, Algeria \\ ${ }^{2}$ Corresponding author \\ E-mail: 1zer35nas@gmail.com, ${ }^{2}$ salah.aguib@yahoo.fr, ${ }^{3}$ abdelkader_nour@hotmail.fr, \\ ${ }^{4}$ djedidtoufik@yahoo.fr, ${ }^{5}$ ali.nedjar@yahoo.fr
}

Received 25 April 2018; accepted 3 May 2018 DOI https://doi.org/10.21595/vp.2018.19934

Check for updates

Copyright (C) 2018 Nassim Zeerouni, et al. This is an open access article distributed under the Creative Commons Attribution License, which permits unrestricted use, distribution, and reproduction in any medium, provided the original work is properly cited.

\begin{abstract}
The purpose of this work is to analyze the nonlinear mechanical behavior of sandwich structures with a magnetorheological elastomer (MRE) core subjected to a permanent magnetic field. A detailed study is first carried out to characterize the mechanical behavior of these structures. The tests were carried out in three-point bending on beams of these complex materials for several distances between supports. An experimental study, of the mechanical behavior response realized using a Zwick $2.5 \mathrm{kN}$ machine, allows to measure displacements as a function of force. The results deduced from the numerical simulation by the Abaqus software are compared with those obtained from the theoretical analysis. This study allows to show that these structures exhibit a non-linear behavior even at small deformations due to the rheological parameters which are more sensitive by the application of a magnetic field.
\end{abstract}

Keywords: sandwich beam, magnetorheological elastomer, magnetic field, three-point bending, smart materials.

\section{Introduction}

Today, it is possible to go further with the magnetorheological materials: controlled by magnetic field, they can change continuously, quickly and reversibly some of their characteristics and adapt to a new environment; the interest of the industry is related to many possible applications, including active damping devices [1]. Zhou and Wang [1, 2] formulated an analytical model of the vibrating motion of a sandwich beam under a uniform magnetic field, perpendicular to the direction of the thickness. To validate the analytical model, they conducted a second study based on numerical modeling to control the rheological properties. Dwivedy et al. [3] studied the parametric instability areas of a sandwich beam with a magnetorheological elastomer core identical to that studied by Zhou and Wang [1,2], but subjected to a periodic load, to determine the adjustment advantages of the mechanical properties of the loaded elastomers exposed to a magnetic field. They used Hsu's method to determine the zones of instabilities associated with single and combined resonance frequencies for different boundary conditions [3]. Nayak et al. [4] studied the vibration reduction of a sandwich beam with three cores, an uncharged elastomer core, a charged elastomer core with ferromagnetic particles, and a charged core with carbon particles. Areas of parametric instability for the two different types of particle charging were studied only for the first three vibration modes to illustrate the passive and active vibration reduction. Sun et al. [5] investigated the controllable capability of an adaptive MRE sandwich beam; the experimental investigations showed that the MRE damps unwanted motion. Hu et al. $[6,7]$ conducted an experimental study on the dynamic response of a sandwich beam containing two aluminum layers with an MRE core under non-homogeneous magnetic fields; it was shown that a reduction of $13.9 \%$ is achieved on the first mode of transverse motion. More recently, the semi-active control of an MRE sandwich beam was investigated under different loadings [8]; the results showed that 
the changing stiffness and damping of the MRE layer controls the motion amplitude.

Based on the above works, the static study and characteristics of bending sandwich beam were investigated in this present paper. By this way, we can evaluate the influence of the magnetic field and the length of the adaptive beam on the amplitude of transverse displacement [9], we have therefore developed an adaptive structure to dampen the amplitude of the displacements in the form of high energy dissipation.

\section{Analytical modeling of bending behavior}

The studied structure is a sandwich beam simply supported with a core made of magnetorheological elastomer (MRE) and two skins made of aluminum such as shown in Fig. 1 [10]. Aluminum skins and MRE, Fig. 2, have different static bending behavior.

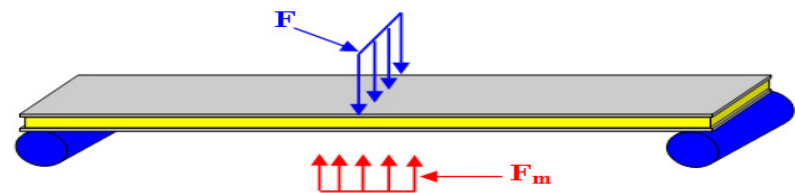

Fig. 1. Sandwich beam in composite material (MRE)
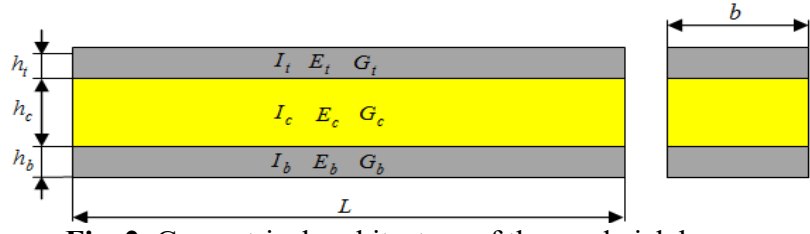

Fig. 2. Geometrical architecture of the sandwich beam

In order to best represent the static behavior of the beam without resorting to complex theories of higher order, the Nilsson model [7], taking some of the principles from the Timoshenko theory [11] will be used. However, Nilsson works were interested in a sandwich structure where skins are homogeneous materials.

In this case, the equilibrium equation, written as a function of the longitudinal elasticity and the shear moduli of the beam, is given by [12]:

$\frac{d M(x)}{d x}-(G A)_{e q}\left(\varphi_{x}+\frac{d w}{d x}\right)=0$.

The integration of Eq. (1) makes it possible to write the expression of the bending:

$w(x)=-\frac{1}{12(E I)_{e q}}\left(F-F_{m}\right) x^{3}+\frac{L^{2}}{16(E I)_{e q}}\left(F-F_{m}\right) x+\frac{1}{2(G A)_{e q}}\left(F-F_{m}\right) x$.

Bending therefore depends on the equivalent rigidity of the beam in bending $(E I)_{e q}$ and in shear $(G A)_{e q}$.

The bending in the center of the beam can be written as:

$w_{\max }\left(\frac{L}{2}\right)=-\left(\frac{L^{3}}{24(E I)_{e q}}+\frac{L}{4(G A)_{e q}}\right)\left(F-F_{m}\right)$,

with: 
$(E I)_{e q}=\frac{1}{12} E_{c} h_{c}+E_{p}\left(\frac{1}{2} h_{c}^{2}+h_{c} h^{2}+\frac{2}{3} h^{3}\right)$,

where: $h_{b}=h_{t}=h$ et $E_{b}=E_{t}=E_{p}$.

And its equivalent shear stiffness $(G A)_{e q}$ is expressed as:

$(G A)_{e q}=\frac{b d^{2} G_{c}}{c} \approx b d G_{c}=A G_{c}$.

\section{Finite element modeling}

The calculation of structures by finite elements is performed using the Abaqus software. The element used is a 2-D element, CPS4 type with four nodes (Fig. 3(a)). The sandwich material is modeled by three structures, two isotropic elastic structures corresponding to the aluminum skins, and an MRE core. The skins are characterized by the Young's modulus, Poisson's ratio and density, given in Table 1. The mechanical and rheological characteristics of the MRE measured experimentally were given by [13]. The sandwich beam model realized in Abaqus is given in Fig. 3(b, c).

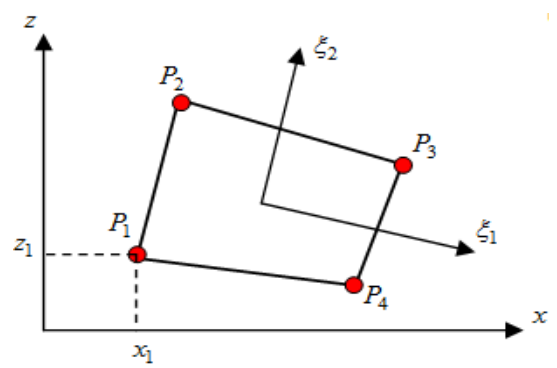

a)

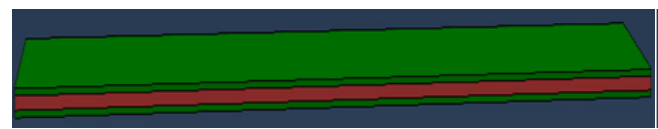

b)

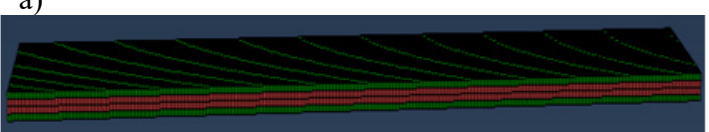

c)

Fig. 3. Sandwich beam model in Abaqus: a) mesh element in Abaqus: 2D type CPS4,

b) beam without meshing, c) beam with meshing
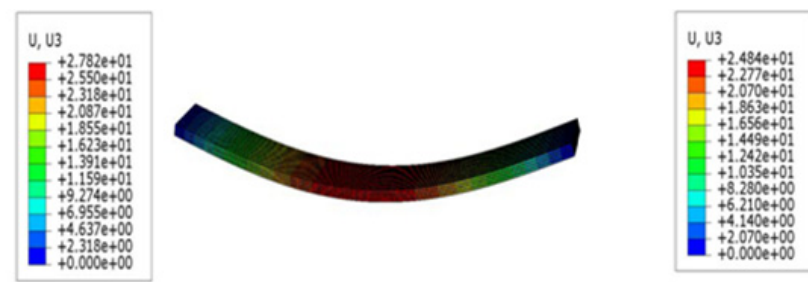

a) $B=0 T$
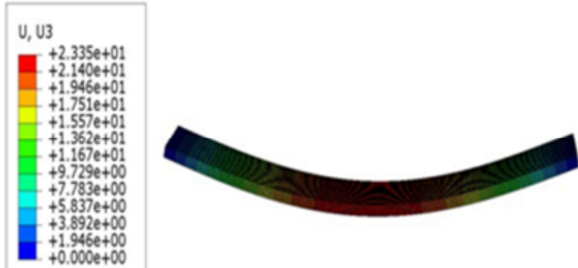

c) $B=0.3 T$

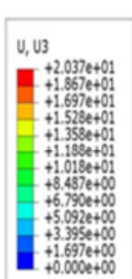

b) $B=0.1 T$
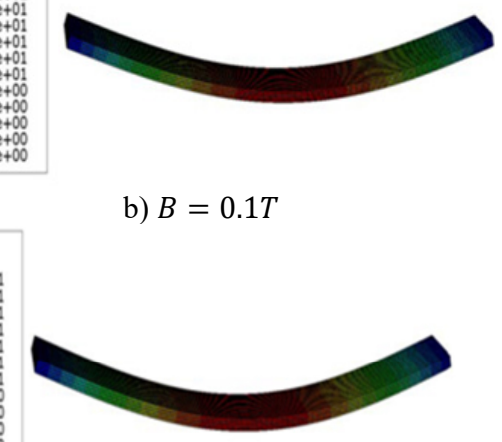

d) $B=0.5 T$

Fig. 4. Deflection of the beam for different values of the magnetic field intensity 
Fig. 4 shows the deflection of the composite material structure for different values of the magnetic field. It is observed that the deflection decreases with the increase of the magnetic field intensity, this is due to the increase in the rigidity of the magnetorheological elastomer.

\section{Experimental analysis}

\subsection{Description of the test}

Sandwich specimens of size $200 \mathrm{~mm} \times 35 \mathrm{~mm} \times 4 \mathrm{~mm}$ are manufactured using two aluminum skins of $1 \mathrm{~mm}$ thickness and an elastomer core charged at $30 \%$ by ferromagnetic particles of micrometric size, which is inserted between the two skins (Fig. 5). The three-point bending test is carried out using a Zwick $2.5 \mathrm{kN}$ machine (Fig. 6). The tests are carried out with a constant deformation rate of $1 \mathrm{~mm} / \mathrm{min}$ in order to be able to consider that the loading is quasi-static. The applied force and the displacements are measured by the cell of the machine.

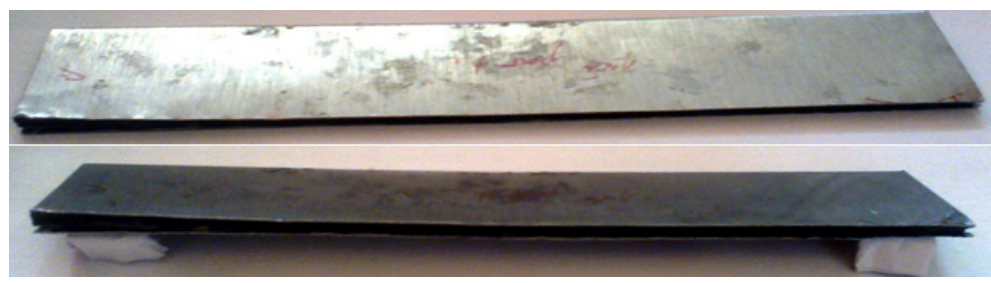

Fig. 5. MRE sandwich specimens

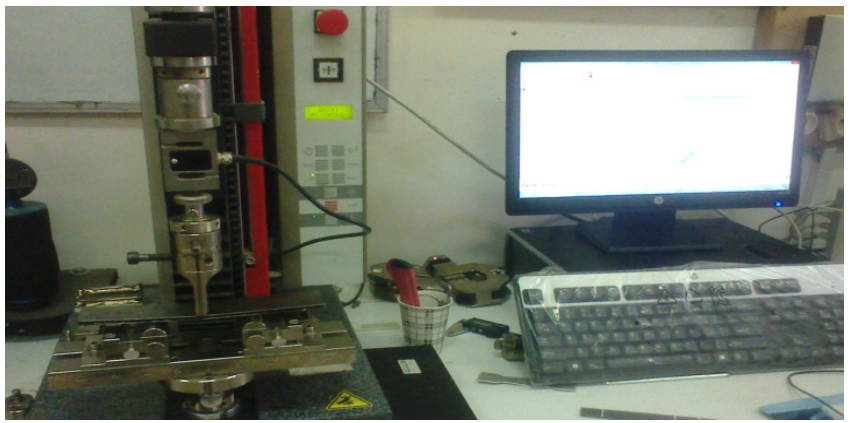

Fig. 6. Three-point bending test machine

\subsection{Interpretation of the results (force/displacement)}

The force-displacement and stress-displacement curves of the beam subjected to a three-point bending load are given in Fig. 7. From these curves, it can be seen that the force at breaking is approximately $115 \mathrm{~N}$ for the test of the piece subjected to a magnetic field intensity of zero. On the other hand, the specimen subjected to a magnetic field intensity of $0.5 T$ is quite far from the rupture. It can be clearly seen that the rupture of the last specimen is not yet pronounced even for the value of the maximum applied force (around $150 \mathrm{~N}$ ) during the test.

\subsection{Interpretation of the results (stress/strain)}

It can be seen from the curves of Fig. 8 that the specimens exhibit a non-linear behavior even at small deformations; it is possible to define distinct domains:

For the test piece subjected to an intensity of $0 T$, we can observe that the behavior is more linear at the beginning of the stress value less than $10 \mathrm{MPa}$, then a non-linear behavior until the rupture. On the other hand, the specimen subjected to an intensity of $0.5 T$ presents a non-linear behavior even with practically negligible strains. 


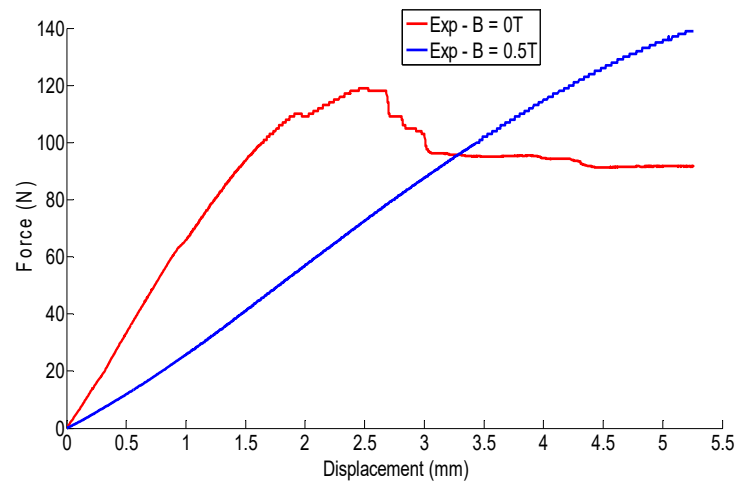

Fig. 7. Three-point bending strength-displacement curve of the sandwich beam with and without magnetic field intensity

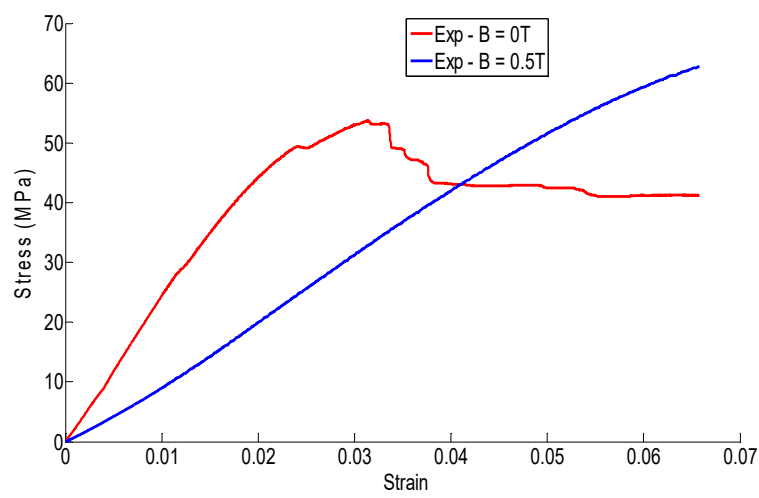

Fig. 8. Three-point bending stress/strain curve of the sandwich beam with and without magnetic field intensity

\section{Conclusions}

The magnetic properties of MRE have been studied by several researches before, but the study on sandwich structures in MRE is new. The MRE manufactured with iron particles dispersed in natural rubber was studied applying magnetic fields varying from 0 to $0.65 T$. The results obtained by the analytical study (Section 2) are compared with those obtained by finite element analysis. The determination of the deflection was performed taking into account the variation of the rheological characteristics of the MRE according to different magnetic field intensities.

The analysis of the results shows that:

1) Overall, the deflection of the beam decreases with the increase of the magnetic field intensity;

2) The results obtained by finite elements and those obtained by analytical study are in good agreement for all magnetic field intensities;

3) The deflection obtained numerically deviates slightly with the increase of the magnetic field intensity of: $6.25 \%$ for $B=0.1 T, 7 \%$ for $B=0.1 T, 9 \%$ for $B=0.5 T$, and $12.25 \%$ for $B=0.65 T$;

4) The evolutions of the deflection as a function of the length of the beam depend on several parameters: the distribution of strain energy between the skins and the core, the magnetic field intensity as well as the elastomer charging rate by the iron particles;

5) The magnetic field intensity has a significant influence on the rigidity of the magnetorheological elastomer;

6) A difference between the analytical values of deflection of the beam and those obtained by 
finite elements analysis appears for high values of the magnetic field intensity. This gap could be explained by the influence of the secondary effects of the magnetic field on certain parameters which are not taken into account by the theoretical study.

\section{References}

[1] Ginder J. M., Nichols M. E., Elie L. D., Tardiff J. L. Magnetorheological elastomers: proprieties and applications. Proceedings of Smart Structures and Materials 1999: Smart Materials Technologies, Vol. 3675, 1999, p. 131-138.

[2] Zhou G. Y., Wang Q. Use of magnetorheological elastomer in an adaptive sandwich beam with conductive skins, Part I: magnetoelastic loads in conductive skins. International Journal of Solids and Structures, Vol. 43, 2006, p. 5386-5402.

[3] Zhou G., Wang Q. Use of magnetorheological elastomer in an adaptive sandwich beam with conductive skins. Part II: dynamic properties. International Journal of Solids and Structures, Vol. 43, 2006, p. 5403-5420.

[4] Nayak B., Dwivedy S. K., Murthy K. S. R. K. Dynamic analysis of magnetorheological elastomerbased sandwich beam with conductive skins under various boundary conditions. Journal of Sound and Vibration, Vol. 330, 2011, p. 1837-1859.

[5] Sun Q., Zhou J.-X., Zhang L. An adaptive beam model and dynamic characteristics of magnetorheological materials. Journal of Sound and Vibration, Vol. 261, 2003, p. 465-481.

[6] Tanju Y., Mergen H. G., Weihua L., Gursel A. Experimental nonlinear dynamics of a geometrically imperfect magneto-rheological elastomer sandwich beam. Composite Structures, Vol. 138, 2016, p. 381-390.

[7] Hu G., Guo M., Li W., Du H., Alici G. Experimental investigation of the vibration characteristics of a magnetorheological elastomer sandwich beam under nonhomogeneous small magnetic fields. Smart Materials and Structures, Vol. 20, 2011, p. 127001.

[8] Dyniewicz B., Bajkowski J. M., Bajer C. I. Semi-active control of a sandwich beam partially filled with magnetorheological elastomer. Mechanical Systems and Signal Processing, Vols. 60-61, 2015, p. 695-705.

[9] Mikhasev G. I., Altenbach H., Korchevskaya E. A. On the influence of the magnetic field on the eigenmodes of thin laminated cylindrical shells containing magnetorheological elastomer. Composite Structures, Vol. 113, 2014, p. 186-196.

[10] Gennadi M., Marina B. Effect of edge shears and diaphragms on buckling of thin laminated medium-length cylindrical shells with low effective shear modulus under external pressure. Acta Mechanica, Vol. 228, Issue 6, 2017, p. 2119-2140.

[11] Yeh J. Y. Vibration analysis of sandwich rectangular plates with magnetorheological elastomer damping treatment. Smart Materials and Structures, Vol. 22, 2013, p. 964-1726.

[12] Friedman Z., Kosmatka J. B. An improved two-node Timoshenko beam finite element. Computers and Structures, Vol. 47, Issue 3, 1993, p. 473-481.

[13] Ying Z. G., Ni Y. Q., Ye S. Q. Stochastic microvibration suppression of a sandwich plate using a magnetorheological viscoelastomer core. Smart Materials and Structures, Vol. 23, 2014, p. 25019. 